\title{
Factores determinantes de la violencia física hacia la mujer en el Perú, periodo 2015-2019
}

Julio Cesar Quispe Mamani jcquispe@unap.edu.pe

Carmen Nievez Quispe Lino cnquispe@unap.edu.pe

Katia Marleny Arpasi Chambi kmarpasi@unap.edu.pe

Veronica Llanos Condori vllanos@unap.edu.pe

Miriam Serezade Hancco Gomez mhancco@unap.edu.pe

Betsy Quispe Quispe betsyquispe@unap.edu.pe Universidad Nacional del Altiplano

\section{RESUMEN}

Puno - Perú

La violencia es un problema que actualmente recibe más denuncias por parte del género femenino, siendo este el más vulnerable en el Perú, por lo que, la presente investigación tiene como objetivo determinar los diferentes factores que intervienen en la violencia física que ocurre en el Perú hacia la mujer. Se consideró un enfoque mixto, de tipo no experimental, donde para el análisis de datos se consideró la encuesta realizada por el Instituto Nacional de Estadística e Informática, ENDES usando el modelo datos de panel con efectos fijos, desde el año 2015 al 2019. Los resultados demuestran que la violencia hacia la mujer no está determinado por la edad ni el número de hijos que estas puedan tener en la familia, tampoco por el estado civil de la persona; por el contrario es explicado por el nivel de estudio en $0.1 \%$, por si la pareja que consume bebidas alcohólicas en $2.6 \%$, si el esposo se pone celoso en $1.26 \%$, por la agresión verbal en $10.46 \%$ y por la agresión emocional en $22.24 \%$. Las conclusiones nos permiten verificar que no todas las variables influyen en la variable dependiente y que existen diversos factores por la que se comete la violencia física, y que necesariamente deberían ser analizadas, además se logra obtener resultados tentativos que ayuden a la sociedad a identificar las razones por las que existe violencia física en el Perú.

Palabras clave: violencia física; violencia doméstica; características sociales; efectos fijos. 


\title{
Determinant factors of physical violence against women in Peru, period 2015-2019
}

\begin{abstract}
Violence is a problem that currently receives more complaints from the female gender, this being the most vulnerable in Peru, therefore, the present research aims to determine the different factors that intervene in the physical violence that occurs in Peru towards the woman. A mixed, non-experimental approach was considered, where for the data analysis the survey carried out by the National Institute of Statistics and Informatics, ENDES, using the panel data model with fixed effects, from 2015 to 2019 was considered. Results show that violence against women is not determined by age or the number of children they may have in the family, nor by the person's marital status; on the contrary, it is explained by the level of study in $0.1 \%$, if the couple who consumes alcoholic beverages in $2.6 \%$, if the husband is jealous in $1.26 \%$, by verbal aggression in $10.46 \%$ and by emotional aggression in $22.24 \%$. The conclusions allow us to verify that not all the variables influence the dependent variable and that there are various factors by which physical violence is committed, and that they should necessarily be analyzed, in addition, tentative results are obtained that help society to identify the reasons why there is physical violence in Peru.
\end{abstract}

Keywords: physical violence; domestic violence; social characteristics; fixed effects.

Artículo recibido: 10 Agosto. 2021 Aceptado para publicación: 07. Setiembre. 2021

Correspondencia: jcquispe@ unap.edu.pe Conflictos de Interés: Ninguna que declarar 


\section{INTRODUCCIÓN}

La violencia física afecta a las mujeres independientemente de su cultura, clase social, etnia, educación y orientación sexual. Existen estudios realizados con un enfoque a nivel mundial, en el cual se manifiesta que al menos el $25 \%$ de las mujeres han sido abusadas (Castillo et al., 2018; Quispe et al., 2018; Trujillo, 2019; Vargas, 2017). Las actitudes de cómo estas situaciones inciertas donde el odio y rechazo hacia la mujer se ha convertido en un arma letal para muchas, es ir hacia aquello que si no logramos identificar tiene graves consecuencias (Jaen et al., 2015; Lorente-Acosta, 2020; Medina-Gamero \& Regalado-Chamorro, 2021). Nos referimos al patriarcado como una manera de demostrar poder (Navarro et al., 2014). Al establecer una determinada fecha o una época de inicio de este sistema político es complejo, pues las diferentes comunidades de homínidos y humanos no se desenvolvían a la par continuamente. Esto es de acuerdo a la antigüedad y ahí inician con los patriarcados esto se debes a los frecuentes debates de corrientes economicistas y políticas, y las arqueológicas y antropológicas (Castillo et al., 2018). En el caso de economistas y políticos el patriarcado vendría de las primeras sociedades como estructura civil y reparto sexual de los haberes, además del nacimiento de la propiedad privada y la familia patriarcal (Durán et al., 2020). La arqueología se remonta en más parámetros históricos porque en la actualidad se pueden observar aun evidencias del poder masculino y el patriciado (Trujillo, 2019).

El concepto de violencia familiar que se referirá en el presente estudio será el mencionado por la Organización Mundial de la Salud que define a nivel global por la Organización mundial de la salud que la violencia contra la mujer es "todo acto de violencia de género que resulte, o pueda tener como resultado un daño físico, sexual o psicológico para la mujer, inclusive las amenazas de tales actos, la coacción o la privación arbitraria de libertad, tanto si se producen en la vida pública como en la privada" (Archer, 2000; Organización Mundial de La Salud, 2020; Pujol \& Mohíno, 2019). Lamentablemente hoy en día la violencia contra las mujeres se podría definir como una pandemia de acciones alarmantes a nivel mundial, se considera como una de las actuaciones que más vulneran y violan a los derechos humanos (Guajardo \& Rivera, 2015; Pacheco, 2013; Vargas, 2017). El análisis más reciente emitido por la Organización Mundial de la Salud (OMS) se señala que a nivel mundial prevalece la violencia física y sexual, y que es ejercida por 
un compañero íntimo entre las mujeres (Desmarais et al., 2012; Gómez Ricárdez et al., 2007; Muñoz \& Echeburúa, 2016).

En América Latina, las encuestas sobre demografía y salud reproductiva, bienestar y educación es estudiado como un carácter comparativo a nivel nacional y regional, que han sido diseñadas hasta la actualidad por la Organización Panamericana de la Salud (OPS), se tiene la muestra que indica que la violencia sexual hacia las mujeres a causa de su acompañante íntimo, y nos indican que la violencia sexual contra las mujeres ejercidas por un compañero íntimo es señalada como una situación común en la sociedad, aunque esto tiene diferencia entre los países desarrollados o sub desarrollados (Dabas, 2001; Navarro et al., 2014; Trujillo, 2019). En el estudio realizado por la OPS mediante encuestas demográficas se tiene un tema preocupante, porque el informe expone que en América Latina y el Caribe que está compuesta por más de 12 países, se visualiza que existe un cantidad considerable de porcentaje en el que se aprecia que las mujeres casadas y unidas con su acompañante íntimo, informan haber estado más expuestas a la violencia física o sexual, por ejemplo en República Dominicana en 2007 se tuvo un 17\% de víctimas haber pasado por una situación similar, en Perú en el 2007-2008 se reflejó que un $39 \%$ sufrió esta tipo de violencia física o sexual. En este contexto se puede afirmar que muchas de las encuestas que se han referido a una conclusión similar, la mitad a la cuarta parte de las mujeres anunciaron haber sido víctimas de una situación parecida donde sus derechos humanos son vulnerados, entonces se afianza que existe más violencia física o sexual por parte del acompañante intimo que por otra persona en relación a la víctima. Asimismo, se pudo visualizar que las situaciones de violencias físicas o sexuales realizadas por parte el acompañante íntimo en términos de porcentaje disminuyeron en los últimos 12 meses, pero este no es un problema que queda absuelto, porque según manifestaciones de las victimas prevalece en un 7,7\% en Jamaica en 2008, 2009 y un 25,5\% en Bolivia 2008 (Guajardo \& Rivera, 2015).

Este problema es visible en la sociedad diariamente, existiendo así en los últimos tiempos revoluciones feministas desde entonces se animó a muchas mujeres a juzgar a sus agresores y aumento las denuncias, aumento su valor de exponer a su agresor suelen advertirnos mediante medios de comunicación, En la mayoría de los países los Estados han sido exigidos por la población a tener participación en este tema, creando así ministerios exclusivamente para mujeres mediante nuevas leyes a favor de las mujeres 
violentadas y también hay otras ya existentes todo esto a favor de la víctima. Según el informe de PNUD y ONU Mujeres Del compromiso a la acción: políticas para erradicar la violencia contra las mujeres en América Latina y el Caribe (Moreno \& Pardo, 2018), en la mayoría de los países de Latinoamérica, existen leyes básicamente para cuidar a la víctima y hacer frente a la violencia doméstica, en muchos países tiene mucha relevancia la violencia y feminicidios y las denuncias fueron realizadas en un juzgado penalmente, es por eso que aun este problema es tratado con indiferencia o sin relevancia en otros Estados. Cabe resaltar que en algunos países las leyes son más rigurosos y castigados con una mayor intensidad, en Brasil el maltrato hacia la mujer está condenado como un castigo atroz; en Chile se tuvo la acción de tratar la violencia intrafamiliar por medio de consultas con la sociedad civil y, finalmente, San Cristóbal y Nieves en estos dos países plasman la violencia sexual y a la violación como un acto de máxima gravedad. (Moreno \& Pardo, 2018)

En el Perú, según la encuesta realizada por el INEI el 32,2\% de las mujeres al menos una vez han sido víctimas de violencia física, verbal o sexual por parte de su pareja sentimental o conyugue, el otro $64,2 \%$ de las mujeres sufren de violencia psicológica y/o verbal y el otro 60,5\% de las mujeres deja al descubierto haber sido víctima de maltrato de al menos una vez en su vida. La violencia sobre género contra las mujeres La tendencia se mantiene y la violencia de género contra las mujeres se ha dispuesto se ha dispuesto como un fenómeno estructural de la sociedad peruana mientras que su tamaño se hace extremadamente difícil, por eso una debe haber una mayor democratización e igualdad de géneros en una relación donde ambos sexos se dividan las tareas, por ejemplo, elegir nuestra sexualidad libremente sin miedo al qué dirán, las elecciones de una carrera profesional o que hacer en nuestra vida cotidiana. Algunos grupos hacer manifestaciones en contra de la violencia de pareja ya que se característica de cada cultura. En el Perú hay una gran variedad de culturas que son resultado de su adecuación a contextos geográficos específicos y están condicionadas por diferentes economías y ecologías. Estas diferencias han dado lugar a una variedad de sistemas culturales de reglas, normas, significados y expectativas compartidas, incluidos los papeles de género, la división sexual del trabajo, la división sexual del poder, las normas sociales de género, la representación pública respecto a la violencia y el honor, entre otros. A través de estos importantes estudios cualitativos realizados en el país se ha expuesto a diferentes tipos de relaciones de pareja 
y violencia física en las poblaciones según las características culturales y sociales específicas (Pacheco, 2013).

Con relación a la prevalencia de violencia familiar, el Programa de Naciones Unidas manifestó el informe en Perú, para insistir que las denuncias realizadas entre los años de 1994 y 1997 en Lima suman alrededor de 20515, para 1997 la cantidad de las denuncias realizadas incremento significativamente en la Primera Comisaría de Mujeres en Lima fue de alrededor de 6000 lo que evidencia un significativo incremento de más de un 300\% con respecto al año anterior. Solo en 1997, del total de denuncias registradas en Lima, el $76.5 \%$ fueron por agresión física se puede decir que 18,801 registradas en Lima, el 76.5\% fueron por violencia física, es decir 18,801 y 5,775 fueron por violencia psicológica, lo que representa un $23.5 \%$. Así mismo, el 74.4\% de los agresores se encontraban ecuánimes y el $24.1 \%$ en estado de ebriedad y $1.5 \%$ drogadas (Vaiz \& Nakano, 2004).

Si no se deja de reconocer la naturaleza multicausal y compleja del fenómeno y la variedad de actitudes que se puedan orientar en la conducta humana, en esta investigación se trata de seleccionar información que los que toman las decisiones puedan usar para pasar a la acción. Así, para nuestra investigación se escogieron, basados en datos y encuestas verídicas, las variables puestas en análisis serán edad, número de hijos, esto civil, ultimo nivel de estudios, pareja que consume bebidas alcohólicas, el esposo se pone celoso con otro hombre, agresión verbal, agresión emocional. Se realizó la categorización de variables y la definición de los ámbitos geográficos sobre la base de estudios cuantitativos y cualitativos nacionales (Vargas, 2017).

El problema central de esta investigación es explicado por los diferentes factores que tienen implicancia en la violencia física que hay en el Perú, en contra de la mujer; toda vez que en los últimos años han incremento en gran proporción los feminicidios, maltratos físicos, psicológicos y sexuales hacia la mujer, por lo que, la violencia es un fenómeno complejo pueden actuar factores culturales, políticos, socioeconómicos, grupales, familiares, ambientales. La pregunta a analizar en la presente investigación fue de ¿Cuáles son los factores determinantes en la violencia física en contra de la mujer?, el objetivo fue identificar la prevalencia de violencia física reciente de pareja y los factores asociados en el Perú, usando la información de la encuesta demográfica 2015- 2019. Las hipótesis a comprobar fueron que, la combinación de los distintos factores de desigualdad produce diferentes grados de violencia contra las mujeres en el Perú, además que la violencia hacia 
la mujer provocada a través del maltrato físico ocasiona significativamente lesiones externas en el cuerpo.

\section{ESTRATEGIAS METODOLÓGICAS O MATERIALES Y MÉTODOS}

Enfoque: Esta investigación tiene un enfoque cuantitativo y cualitativo ya que las variables son manipulables de acuerdo a la conveniencia de la investigación científica, recopilando datos por medio del INEI (ENDES) (Hernández et al., 2010).

Diseño de la investigación: El diseño de nuestra investigación es no experimental usando el modelo de regresión con datos de panel y efectos fijos aplicando datos de corte transversal y series de tiempo desde los años 2015-2019. Ya que recolectamos datos de una encuesta del instituto nacional de estadística e informática ENDES (Mendoza, 2014) Métodos: Esta investigación corresponde al método descriptivo ya que se analizó las encuestas así podemos obtener nuestras variables sobre la violencia física a la mujer (Cazau, 2006).

Técnicas: Se utilizó un cuestionario de encuestas con el fin de buscar, explorar y hacer una recolección de datos mediante preguntas formulada por el instituto nacional de estadísticas e informáticas ENDES.

Población y muestra: La población objetivo según la encuesta demografía y salud pública, los hogares fueron considerados particulares y a todos sus miembros, también personas que son residentes concurrentes habituales y también los no residentes. Las mujeres encuestadas varían desde los 12 a 49 años de edad, una persona de 15 años a más de edad por cada hogar particular, se considera un dominio anual, los datos se encuentran en el módulo Mortalidad materna y violencia familiar.(INEI (ENDES), n.d.)

Para la muestra de esta investigación se consideró ser bietápica, probabilística de tipo equilibrado, estratificada e independiente y auto ponderado a nivel departamental y por área Urbana y Rural. Para el año 2015 se encuesto 359,000 viviendas, 14,140 viviendas pertenecientes a capitales de departamento y distritos de Lima Metropolitana, 9,310 viviendas pertenecientes al resto de urbano y 12,450 viviendas pertenecientes al área rural.

Para el año 2016 el tamaño de la muestra de la ENDES es de 35,910 viviendas, 14,160 viviendas pertenecientes a capitales de departamento y distritos de Lima, 9,310 viviendas pertenecientes al resto de urbano y 12,450 viviendas pertenecientes al área rural. Para el año 2017 el tamaño de la muestra de la ENDES es de 35,910 viviendas, 14,160 viviendas 
pertenecientes a capitales de departamento y 43 distritos de Lima Provincia, 9,310 viviendas pertenecientes al resto de urbano y 12,450 viviendas pertenecientes al área rural.

Para el año 2018 el tamaño de la muestra de la ENDES es de 36,760 viviendas, 14,760 viviendas pertenecientes a capitales de departamento y 43 distritos de Lima Provincia, 9,340 viviendas pertenecientes al resto de urbano y 12,660 viviendas pertenecientes al área rural. Para el año 2019 el tamaño de la muestra de la ENDES es de 36,760 viviendas, 14,780 viviendas pertenecientes a capitales de departamento y 43 distritos de Lima Provincia, 9,320 viviendas pertenecientes al resto de urbano y 12,660 viviendas pertenecientes al área rural.

Análisis de variables: Las variables en estudio para esta investigación obtenidos de ENDES 2015-2019, las variables descritas son: violencia física, edad, número de hijos, esto civil, ultimo nivel de estudios, pareja que consume bebidas alcohólicas, el esposo se pone celoso con otro hombre, agresión verbal, agresión emocional (Tabla 1).

Tabla 1. Características de las variables

\begin{tabular}{lcccc}
\hline \multicolumn{1}{c}{ Variables } & Notación & \multicolumn{1}{c}{ Concepto } & Tipo & $\begin{array}{c}\text { Unidad de } \\
\text { medida }\end{array}$ \\
\hline Dependiente & & & & \\
\hline Violencia física & Vfisica & $\begin{array}{l}\text { Acción que puede ocasionar daño, } \\
\text { utilizando la fuerza física o alguna } \\
\text { clase de armamento u objeto que } \\
\text { pueda causar lesiones. }\end{array}$ & Cualitativo & $\begin{array}{c}\text { Unidad por } \\
\text { persona }\end{array}$ \\
\hline Independientes & edad & Tiempo que vivió una persona. & Cuantitativo & Años \\
\hline Edad & numhijos & $\begin{array}{l}\text { El número de hijos que la mujer ha } \\
\text { concebido. }\end{array}$ & Cuantitativo & $\begin{array}{c}\text { Cantidad de } \\
\text { hijo }\end{array}$ \\
\hline $\begin{array}{l}\text { Número de hijos } \\
\text { Estado civil }\end{array}$ & ecivil & $\begin{array}{l}\text { Situación en la que una persona se } \\
\text { encuentra en un determinado } \\
\text { tiempo de su vida. }\end{array}$ & Cualitativo & $\begin{array}{c}\text { Por estado } \\
\text { conyugal }\end{array}$ \\
\hline $\begin{array}{l}\text { Ultimo nivel de } \\
\text { estudios }\end{array}$ & nivestu & $\begin{array}{l}\text { El grado más alto de estudios que } \\
\text { una persona haya cursado. }\end{array}$ & Cualitativos & $\begin{array}{c}\text { Grado } \\
\text { académico }\end{array}$ \\
\hline $\begin{array}{l}\text { Pareja que } \\
\text { consume bebidas } \\
\text { alcohólicas }\end{array}$ & parconbeba & $\begin{array}{l}\text { Consumo de bebidas alcohólicas } \\
\text { en la relación familiar. }\end{array}$ & Cualitativo & Proporción \\
\hline $\begin{array}{l}\text { El esposo se pone } \\
\text { celoso si habla con } \\
\text { otro hombre }\end{array}$ & celos & $\begin{array}{l}\text { La idealización de la pareja de } \\
\text { no saber dónde estás o qué estás } \\
\text { haciendo. }\end{array}$ & Cuantitativo & Nivel \\
\hline & & & & \\
\hline
\end{tabular}


Se caracteriza porque pretende Agresión verbal agreve hacer daño a otra persona con un mensaje o un discurso Cualitativo Proporción hiriente.

Agresión emocional

agreemo Abuso mental o psicológico.

Cualitativo Proporción

\section{Fuente: Elaboración propia}

\section{RESULTADOS Y DISCUSIÓN}

\section{Comportamiento de la violencia física 2015-2019}

Se visualiza en grafico de la violencia física de la mujer año 2015, de las mujeres encuestadas que en un porcentaje de $89.69 \%$ no sufrieron violencia física y el restante de $11.31 \%$ fueron víctimas de algún acto violento (Figura 1).

\section{Figura 1. Porcentaje de violencia física contra la mujer año 2015}

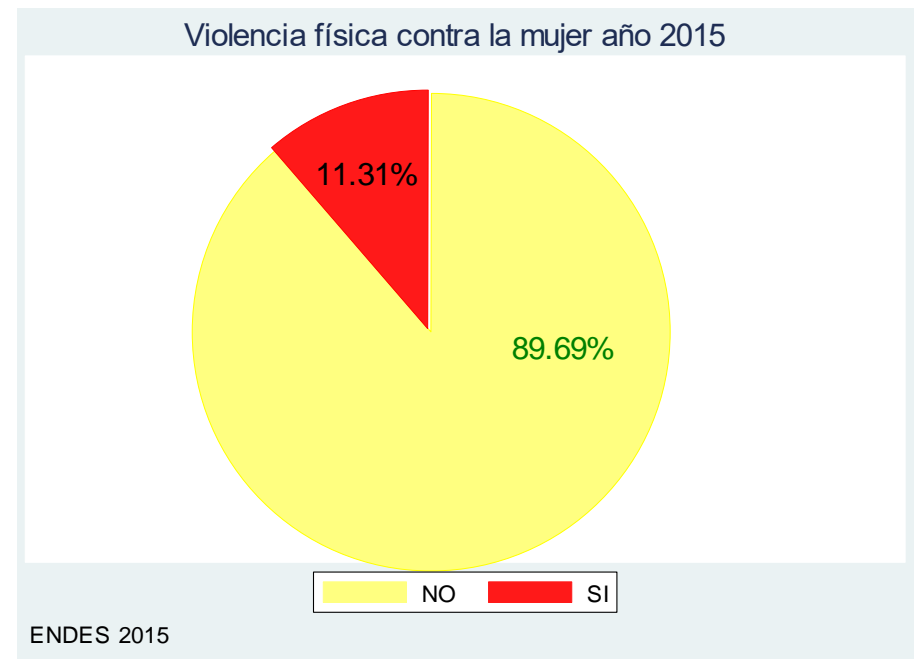

Fuente: elaboración propia

Se analiza en gráfico de la violencia física de la mujer año 2016, de la población de las mujeres encuestadas que en un porcentaje de $89.16 \%$ manifiestan no haber sufrido de violencia física y el restante de $10.84 \%$ fueron víctimas de algún acto violento (Figura 2).

Figura 2. Porcentaje de violencia física contra la mujer año 2016 


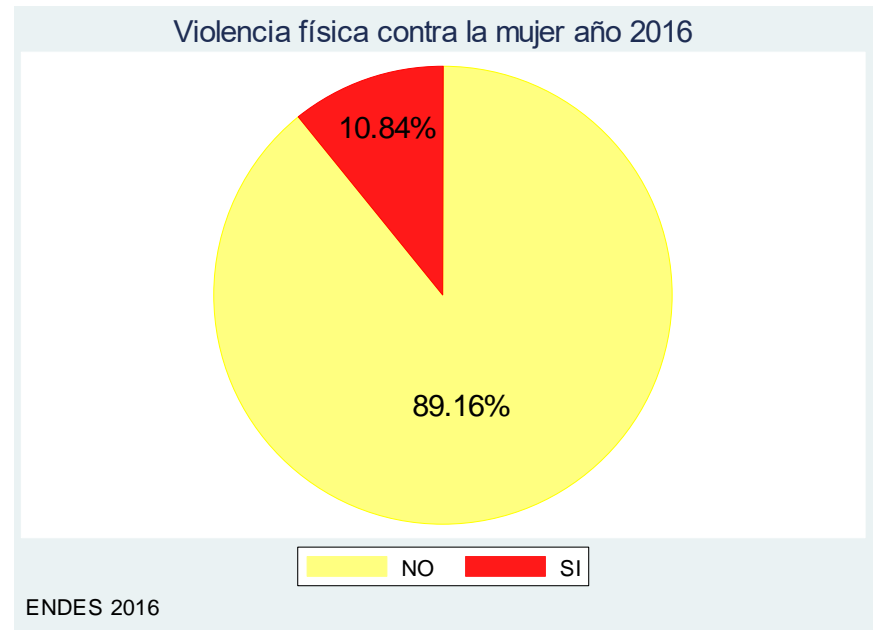

Fuente: elaboración propia

Se determina en el siguiente grafico de la violencia física de la mujer año 2017, del total de las mujeres encuestadas un porcentaje de $89.30 \%$ no sufrieron violencia física siendo menos que el año anterior y el restante de $10.30 \%$ fueron víctimas de algún acto violento (Figura 3).

Figura 3. Porcentaje de violencia física contra la mujer año 2017

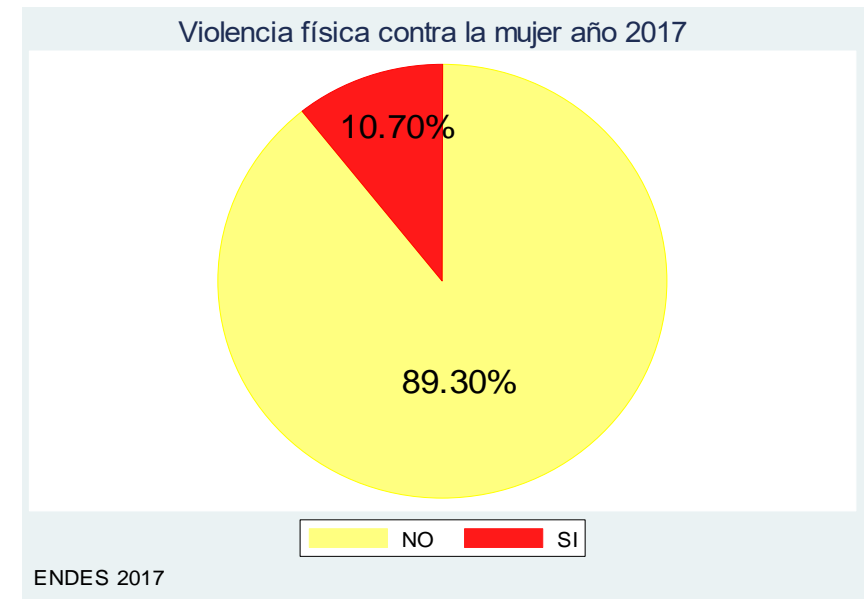

Fuente: elaboración propia

En el siguiente grafico de la violencia física de la mujer año 2018, de las mujeres encuestadas que en un porcentaje de $89.53 \%$ no sufrieron violencia física aumentando para este año un porcentaje minino en comparación del año anterior y el restante de $10.47 \%$ fueron víctimas de algún acto violento (Figura 4).

Figura 4. Porcentaje de violencia física contra la mujer año 2018 


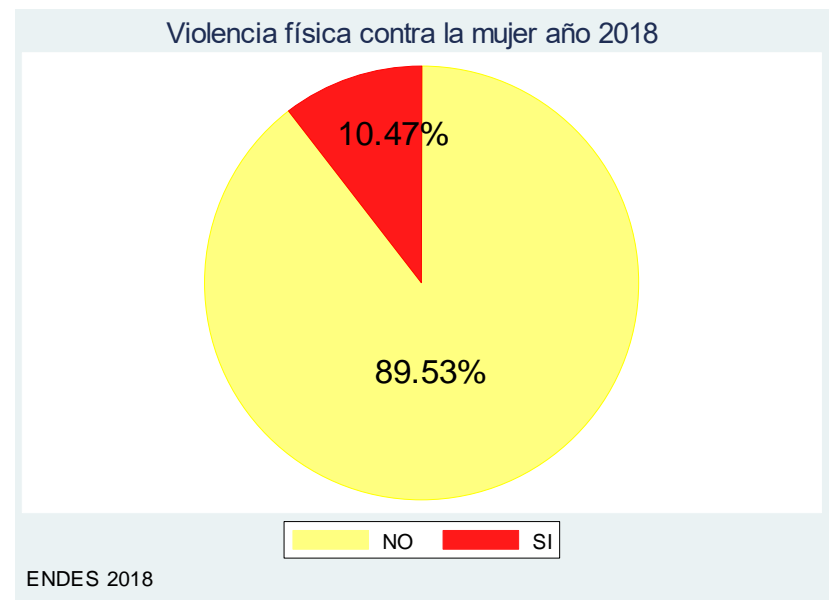

\section{Fuente: Elaborarían propia}

En el posterior gráfico de la violencia física de la mujer año 2019, de las mujeres encuestadas que en un porcentaje de $89.85 \%$ no sufrieron violencia física y el restante de $10.15 \%$ fueron víctimas de algún acto violento (Figura 5).

Figura 5. Porcentaje de violencia física contra la mujer año 2019

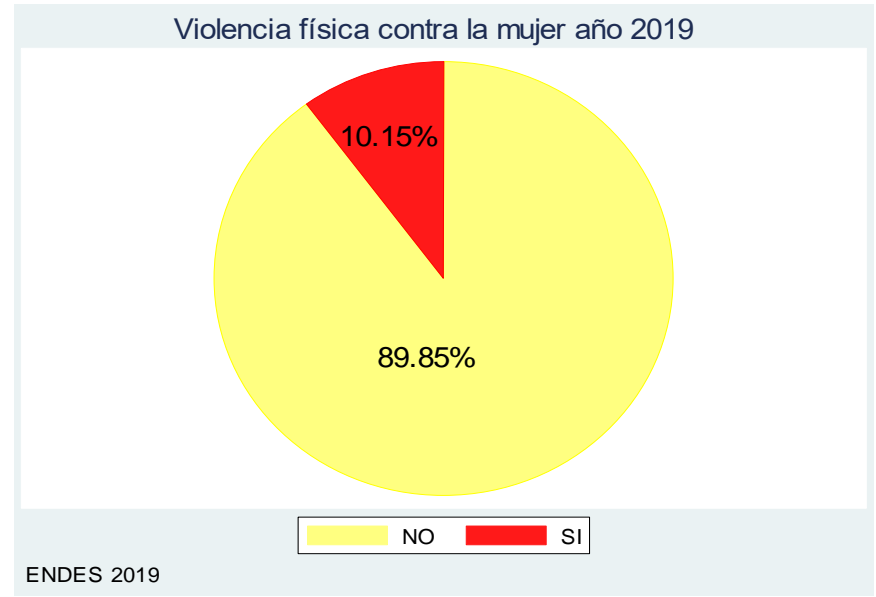

Fuente: Elaborarían propia

\section{Análisis descriptivo de las variables determinantes de la violencia de la mujer}

Al analizar respecto al estado civil de las mujeres encuestadas, existe una mayor porcentaje $28.22 \%$ de mujeres que conviven con sus parejas, seguido de un $25.95 \%$ de mujeres que son casadas, un $24.75 \%$ de mujeres solteras, con porcentajes menores de $8.96 \%$ mujeres separadas, $7.32 \%$ de mujeres viudas y con aun menor porcentaje de $4.79 \%$ de mujeres divorciadas (Tabla 2). 
Tabla 2. Resultados de encuesta ENDES estado civil.

\begin{tabular}{lcc}
\hline \multicolumn{1}{c}{ Estado civil } & Frecuencia & Porcentaje \\
\hline Soltero & 26,134 & 24.75 \\
Casado & 27,400 & 25.95 \\
Conviviente & 29,797 & 28.22 \\
Viudo & 7,725 & 7.32 \\
Divorciado & 5,059 & 4.79 \\
Separado & 9,460 & 8.96 \\
\hline \multicolumn{1}{c}{ Total } & $\mathbf{1 0 5 , 5 7 5}$ & $\mathbf{1 0 0 . 0 0}$ \\
\hline
\end{tabular}

Fuente: Elaboración propia

En el caso del nivel de estudios de las mujeres encuestadas, la mayor parte tiene como primaria incompleta con un porcentaje del $22.47 \%$ seguido de los estudios superiores con un porcentaje del $18.42 \%$ como se observa el porcentaje más bajo es de primaria completa (Tabla 3).

Tabla 3. Resultados de encuesta ENDES ultimo nivel de estudios

\begin{tabular}{lcc}
\hline Ultimo nivel de estudios & Frecuencia & Porcentaje \\
\hline Sin educación & 18,036 & 17.08 \\
Primaria incompleta & 23,727 & 22.47 \\
Primaria completa & 7,856 & 7.44 \\
Secundaria incompleta & 17,244 & 16.33 \\
Secundaria completa & 19,048 & 18.04 \\
Superior & 19,444 & 18.42 \\
No sabe & 220 & 0.21 \\
\hline \multicolumn{1}{c}{ Total } & $\mathbf{1 0 5 , 5 7 5}$ & $\mathbf{1 0 0 . 0 0}$ \\
\hline
\end{tabular}

Fuente: Elaboración propia

Realizando el análisis respecto a que si la pareja consume bebidas alcohólicas de las personas encuestadas se muestra un mayor porcentaje de que si consumes bebidas alcohólicas el $69.31 \%$ y el resto del porcentaje $30.68 \%$ no consume bebidas alcohólicas (Tabla 4). 
Tabla 4. Resultados de encuesta ENDES pareja que consume bebidas alcohólicas

\begin{tabular}{|c|c|c|}
\hline $\begin{array}{c}\text { Pareja que consume } \\
\text { bebidas alcohólicas }\end{array}$ & Frecuencia & Porcentaje \\
\hline No & 32,395 & 30.68 \\
\hline $\mathrm{Si}$ & 73,180 & 69.32 \\
\hline Total & 105,575 & 100.00 \\
\hline
\end{tabular}

\section{Fuente: Elaboración propia}

En este caso el hombre se pone celoso de otro hombre, la mayor parte de sus esposos no son celosos con un porcentaje del $62.46 \%$ y el caso de que cuando su esposo si se pone celoso con un porcentaje del $36.95 \%$ y el mínimo no sabe con un porcentaje de $0.59 \%$ (Tabla 5).

Tabla 5. Resultados de encuesta ENDES el esposo se pone celoso de otro hombre

\begin{tabular}{|c|c|c|}
\hline $\begin{array}{c}\text { El esposo se pone celoso } \\
\text { de otro hombre }\end{array}$ & Frecuencia & Porcentaje \\
\hline $0 \mathrm{No}$ & 65,945 & 62.46 \\
\hline $1 \mathrm{Si}$ & 39,006 & 36.95 \\
\hline 8 No sabe & 624 & 0.59 \\
\hline Total & 105,575 & 100.00 \\
\hline
\end{tabular}

Fuente: Elaboración propia

Analizando la agresión verbal cometido contra las mujeres encuestaos, se verifica que con una mayor proporción de $91.31 \%$ de mujeres encuestadas no son víctimas de agresión verbal, el 4.04\% confiesa que nunca fueron víctimas de este acto, el 3.46\% manifiesta que sucede algunas veces y el 1.08\% manifiesta que si ocurre frecuentemente este acto (Tabla 6).

Tabla 6. Resultados de encuesta ENDES agresión verbal

\begin{tabular}{lcc}
\hline \multicolumn{1}{c}{ Agresión verbal } & Frecuencia & Porcentaje \\
\hline No & 96,405 & 91.31 \\
Frecuentemente & 1,144 & 1.08 \\
Algunas veces & 3,653 & 3.46 \\
Nunca & 4,368 & 4.14 \\
No respondió & 5 & 0 \\
\hline \multicolumn{1}{c}{ Total } & $\mathbf{1 0 5 , 5 7 5}$ & $\mathbf{1 0 0 . 0 0}$ \\
\hline
\end{tabular}

Fuente: Elaboración propia 
En el caso del acto de agresión emocional el $75.21 \%$ de las mujeres encuestadas, en su mayoría manifiesta que no son víctimas de este acto, y el restante el $24.79 \%$ manifiestan ser víctimas de agresión emocional (Tabla 7).

Tabla 7. Resultados de encuesta ENDES agresión emocional

\begin{tabular}{|c|c|c|}
\hline Agresión emocional & Frecuencia & Porcentaje \\
\hline No & 79,401 & 75.21 \\
\hline $\mathrm{Si}$ & 26,174 & 24.79 \\
\hline Total & 105,575 & 100.00 \\
\hline
\end{tabular}

Fuente: Elaboración propia

\section{Prueba de hipótesis de investigación}

Se contrastó la prueba de hipótesis general mediante la regresión de efectos fijos en la siguiente tabla.

Tabla 8. Resultados de estimación de efectos fijos

\begin{tabular}{|c|c|c|c|c|}
\hline \multicolumn{3}{|l|}{ R-cuadrado = } & & 0.230742 \\
\hline \multicolumn{3}{|c|}{ R-cuadrado ajustado } & \multicolumn{2}{|r|}{0.230691} \\
\hline \multicolumn{3}{|l|}{ F-estadístico } & \multicolumn{2}{|r|}{4523.617} \\
\hline \multicolumn{3}{|c|}{ Prob(F-estadístico) } & \multicolumn{2}{|r|}{0.00000} \\
\hline \multicolumn{3}{|c|}{ Estadistico Durbin-Watson } & \multicolumn{2}{|r|}{1.972029} \\
\hline Variable & Coeficiente & Error estándar & $\mathbf{z}$ & $\mathbf{p}>[\mathbf{z}]$ \\
\hline Edad & 0.00001 & 0.00004 & 0.27938 & 0.7800 \\
\hline Numhijos & 0.00047 & 0.00049 & 0.95811 & 0.3380 \\
\hline Ecivil & -0.00021 & 0.00058 & -0.36642 & 0.7141 \\
\hline Nivestu & 0.00134 & 0.00049 & 2.70977 & 0.0067 \\
\hline Parconbeba & 0.02649 & 0.00188 & 14.13076 & 0.0000 \\
\hline Celos & 0.01269 & 0.00113 & 11.27292 & 0.0000 \\
\hline Agrever & 0.10468 & 0.00134 & 78.22173 & 0.0000 \\
\hline Agreemo & 0.22249 & 0.00223 & 99.90255 & 0.0000 \\
\hline Constante & 0.00253 & 0.00272 & 0.93004 & 0.3524 \\
\hline
\end{tabular}

Fuente: Elaboración propia 
La interpretación del R-cuadrado ajustado indica que aproximadamente el $23.07 \%$ de la violencia física contra la mujer (vfisica) puede ser explicada por las variables independientes del modelo (edad, número de hijos, esto civil, ultimo nivel de estudios, pareja que consume bebidas alcohólicas, el esposo se pone celoso con otro hombre, agresión verbal, agresión emocional).

También se obtiene un estadístico global $\mathrm{F}=4523.617$ alto, por lo que se rechaza la hipótesis nula y se acepta la hipótesis alterna de que al menos hay una variable que influye en nuestro modelo, entonces la influencia que tienen las variables independientes sobre la variable dependiente son significativas.

Además la regresión realizada no presenta problemas de auto correlación porque se tiene Se determina que 5 de las variables regresionadas en este estudio son significativos para explicar a la violencia física, observando sus probabilidades individuales que son menores al 5\% del nivel de significancia consideradas en este estudio son estadísticamente significativas, por lo tanto si influyen de manera directa en la variable dependiente violencia física.

La violencia hacia la mujer no está determinado por la edad ni el número de hijos que estas puedan tener en la familia, tampoco por el estado civil de la persona; por el contrario, el nivel de estudio si influye en la violencia de la mujer, en vista que si aumenta el nivel de estudio en 1 nivel, entonces la violencia familiar incrementa en $0.1 \%$, es decir es mínimo su impacto. En el caso de si la pareja que consume bebidas alcohólicas, en este caso esta variable es muy significativa, en vista que si aumenta el consumo de bebidas alcohólicas por parte de la pareja, entonces la violencia hacia la mujer incrementa en $2.6 \%$, demostrándose que esto afecta directamente a los integrantes de la familia.

Al considerar la variable si el esposo se pone celoso si habla con otro hombre, esta también es significativa, por lo que si aumenta los celos de la pareja al hablar la mujer con otro hombre, entonces la probabilidad de que se genere la violencia hacia la mujer incrementa en $1.26 \%$, afectando directamente hacia la relación de la pareja. En el caso de la variable de agresión verbal, es significativa, por lo que si se genera una agresión verbal entre la pareja, entonces la probabilidad de que se de la violencia hacia la mujer aumenta en $10.46 \%$. Finalmente la variable agresión emocional es significativa, por lo que si aumenta la agresión emocional en la pareja, entonces la probabilidad de que se genere la violencia en la mujer incrementa $22.24 \%$, cuestión que es coherente y necesario 
tomar en consideración para el establecimiento de políticas y acciones en el hogar y la familia.

Por lo que, evidenciando y buscando realizar la discusión con otros autores, se puede establecer que tiene mucha relación con el reporte de Vargas (2017), donde la relación del agresor con la violencia física de la mujer es considerable, el $24.9 \%$ corresponde a que el causante es el esposo o la pareja, además de acuerdo a la edad, el $87 \%$ de agresores que maltrataban a sus víctimas, se encontraba entre los 15 y 49 años; el 72,7\% era víctima de violencia física y el 12,6\% de violencia psicológica, y los motivos de la agresión fueron los celos $(26,2 \%)$, problemas familiares $(38,9 \%)$ y problemas económicos $(11,9 \%)$; el 4,5\% padeció de agresión sexual. En cambio los resultados obtenidos para esta investigación fueron que las parejas que consumen bebías alcohólicas son más las parejas que consumen bebías alcohólicas con un $69.31 \%$, y además la edad no es una variable que pueda afectar a la violencia física, por el tema de los celos la agresión puede alcanzar un porcentaje mínimo de $36.95 \%$, tato la agresión verbal como la agresión emocional son factores importantes que determinan la violencia física. Y en cambio los resultados para el investigador es que el 59,3\% de los entrevistados se encontraba sobrio en el momento de la agresión, 35,6\% reportó ingesta de alcohol y el 4,6\% alcohol y otras sustancias psicoactivas. Esto confirma lo revelado en otros estudios que vinculan el abuso de sustancias psicoactivas con la conducta violenta.

Según la encuesta ENDES en el año 2016 la violencia física contra la mujer se incrementa con la edad y en quienes dejaron de tener vínculo conyugal. Este hecho también fue observado en este estudio pues las adultas mayores se constituyeron en el segundo grupo de edad que tiene mayor riesgo de sufrir feminicidio. Sin embargo, las niñas ostentaron el mayor riesgo de muerte, por lo que superaron a las adultas mayores. Según Piñeiro, 19 a menor edad se incrementa el riesgo de fatalidad por violencia, debida a su mayor fragilidad; hecha que también sucede en los adultos mayores por la disminución de la fuerza, resistencia y funciones fisiológicas. E manera contraria en este estudio se determina que la edad no tiene influencia en la violencia física de la puesto que la variable no es estadísticamente significativa, además existen muchas otros variables que pueden determinar las causa de la violencia física contra la mujer (Quispe Ilanzo et al., 2018). Finalmente, de acuerdo a los resultados obtenidos según Castillo et al. (2018) en este de investigación se observó que el tipo más frecuente de violencia fue la física con 38,2 \%; 
mientras que el 29,1\%; 14,6 \% y 18,1 \% correspondieron a la psicológica, sexual y económica respectivamente. (Castillo Saavedra et al., 2018) Pero se demuestra que la variable violencia física a lo largo de los años desde 2015 hasta el 2019 no tiene disminución y aumento se mantiene constante por lo que las políticas leyes o normas aplicadas no son efectivas para combatir este problema social En este sentido, los resultados obtenidos revelarían que la violencia de género surge del comportamiento social enseñado o por múltiples factores sociales que permiten la continuidad de este tipo de agresiones, repercutiendo de esta manera, en la salud y en el bienestar físico de la mujer.

\section{CONCLUSIÓN O CONSIDERACIONES FINALES}

La combinación de los distintos factores de desigualdad, las variables consideraras no explican a la violencia física en su totalidad o mayoría ya que se ha observado que solo influye en un $23 \%$ a la violencia física, por lo tanto, demuestra que hay otras variables que explican mejor a la variable violencia física. En el modelo de la regresión con datos de panel con efectos aleatorios no se pudieron regresionar, porque en la prueba de Hausman se rechazaba la hipótesis nula y se aceptaba la hipótesis alterna de que a la variable dependiente el método que mejor se ajusta es el de efectos fijos.

Las conclusiones para esta investigación es que la violencia hacia la mujer por parte de su pareja es una manera de discriminación que causa sufrimientos, dolor y temor ya que afecta a la salud mental como emocional y psíquica de la agraviada que la hace sentir incapaz de poder volver a empezar una nueva vida si no que también afectan a los hijos adolescentes porque también pueden ser víctimas de maltratos en algún momento de su vida y esto les afecta de manera negativa.

La violencia física presentada en todas sus formas como son el feminicidio, acoso sexual, violencia física, violencia psicológica entre otros las variables tomadas de la encuesta, 5 variables son estadísticamente significativas ya que la probabilidad es menor al $5 \%$, también se ha observado que cada año la violencia física ha ido disminuyendo ,para el año 2015 hay un porcentaje de $11.31 \%$ de que si sufrieron violencia física, para el año 2016 hubo un porcentaje de 10,84\%, el 2017 obtuvo un porcentaje de 10,70\%, para el año 2018 hubo un porcentaje de 10,47\% y el ultimo año 2018 tuvo un porcentaje del 10,15\%, esto quiere decir que la violencia física ha disminuido en un porcentaje bajo debido a las sanciones aplicadas por el estado hacia la pareja sentimental de la mujer ya que en los 
últimos años se tomó en cuenta los derechos de la mujer habiendo múltiples denuncias por ende reduce la violencia física hacia la mujer aunque no es de manera efectiva.

Muchas veces las mujeres defienden a su pareja por miedo a que la violencia continúe, también por la sustentación económica, por los malos comentarios de la sociedad y es por ese motivo que se debería investigar más estudios para conocer con mayor profundidad sobre la violencia contra la mujer que es infligida por su pareja o conyugue, a pesar de que el porcentaje de violencia física cometida en el Perú es mínima como se contrasta en este estudio, es un problema que no debería dejar de ser importante para la sociedad además de que se debe crear más leyes y normas a favor de la víctimas para que se sientan seguras y no volver a sufrir un acto de violencia.

Finalmente, la violencia familiar no está determinado por la edad ni el número de hijos que estas puedan tener en la familia, tampoco por el estado civil de la persona; por el contrario, el nivel de estudio si influye en la violencia de la mujer en $0.1 \%$, si la pareja que consume bebidas alcohólicas es muy significativa y explica a la violencia de la mujer en $2.6 \%$. La variable si el esposo se pone celoso es significativa y explica a la violencia de la mujer en $1.26 \%$, la agresión verbal es significativa y explica a la violencia de la mujer en $10.46 \%$ y la agresión emocional es significativa y explica a la violencia de la mujer en $22.24 \%$.

\section{LISTA DE REFERENCIAS}

Archer, J. (2000). Sex differences in aggression between heterosexual partners: A metaanalytic review. Psychological Bulletin, 126(5), 651-680. https://doi.org/10.1037/0033-2909.126.5.651

Castillo Saavedra, E. F., Bernardo Trujillo, J. V., \& Medina Reyes, M. A. (2018). Violencia de género y autoestima de mujeres del centro poblado Huanja - Huaraz, 2017. Horizonte Médico (Lima), 18(2), 47-52. https://doi.org/10.24265/HORIZMED.2018.V18N2.08

Cazau, P. (2006). Introducción a la investigación en ciencias sociales (Tercera Ed). http://www.academia.edu/download/37844523/cazau_-_metodologia.pdf

Dabas, E. (2001). Redes sociales: niveles de abordaje en la intervención y organización en red. http://148.202.167.116:8080/xmlui/handle/123456789/953

Desmarais, S. L., Reeves, K. A., Nicholls, T. L., Telford, R. P., \& Fiebert, M. S. (2012). Prevalence of Physical Violence in Intimate Relationships, Part 1: Rates of Male 
and Female Victimization. Partner Abuse, 3(2), 140-169. https://doi.org/10.1891/1946-6560.3.2.140

Durán, G., Bayón Jiménez, M., \& Bonilla, A. (2020). Habitar ante la cotidianidad de la contaminación del agua: contestaciones a las actividades extractivas en las periferias urbanas de Ecuador. Revistas.Uniandes.Edu.Co, 2020(39), 17-39. https://doi.org/10.7440/antipoda39.2020.02

Gómez Ricárdez, L. A., Abrego, G. R., \& Llamas, E. K. (2007). Prevalencia y factores asociados a violencia familiar en adultos mayores de Ocozocoautla (Chiapas, México). Revista Española de Geriatría y Gerontología, 42(1), 27-34. https://doi.org/10.1016/S0211-139X(07)73518-7

Guajardo Soto, G., \& Rivera Viedma, C. (2015). Violencia contra las mujeres.

Hernández Sampieri, R., Fernández Collado, C., \& Baptista Lucio, P. (2010). Metodología de la investigación (Issue 1). https://doi.org/10.16309/j.cnki.issn.1007-1776.2003.03.004

INEI (ENDES). (n.d.). PERÚ Instituto Nacional de Estadística e Informática.

Jaen Cortés, C. I., Aragón, S. R., Amorin de Castro, E. F., \& Rivera Rivera, L. (2015). Violencia de Pareja en Mujeres: Prevalencia y Factores Asociados. Acta de Investigación Psicológica, 5(3), 2224-2239. https://doi.org/10.1016/S20074719(16)30012-6

Lorente-Acosta, M. (2020). Violencia de género en tiempos de pandemia y confinamiento. Revista Española de Medicina Legal, 46(3), 139-145. https://doi.org/10.1016/J.REML.2020.05.005

Medina-Gamero, A., \& Regalado-Chamorro, M. (2021). Pandemic, confinement and gender violence: A dangerous trinomial. Atencion Primaria, 53(10).

Mendoza Bellido, W. (2014). Cómo investigan los economístas? Guía para elaborar y desarrollar un proyecto de investigación. https://files.pucp.education/departamento/economia/lde-2014-05.pdf

Moreno, R., \& Pardo, L. (2018). La violencia contra las mujeres en latinoamerica. FORREING AFFAIRS.

Muñoz, J. M., \& Echeburúa, E. (2016). Diferentes modalidades de violencia en la relación de pareja: implicaciones para la evaluación psicológica forense en el contexto legal español. Anuario de Psicología Jurídica, 26(1), 2-12. 
https://doi.org/10.1016/J.APJ.2015.10.001

Navarro, D. M., Robles, J. N., \& Hernández, L. O. (2014). La mujer en México: inequidad, pobreza y violencia. Revista Mexicana de Ciencias Políticas y Sociales, 59(220), 117-146. https://doi.org/10.1016/S0185-1918(14)70803-7

Organización mundial de la salud. (2020). Violencia Contra La Mujer.

Pacheco Arevalo, B. E. (2013). el feminicidio y la violencia de genero en la provincia de ocaña, norte de santander, entre los años 2004-2011: analisis social de la comunidad y la normativa imperante en colombia.

Pujol Robinat, A., \& Mohíno Justes, S. (2019). Intimate partner violence and mental illness. Revista Espanola de Medicina Legal, 45(2), 77-82.

Quispe Ilanzo, M. P., Urbano Curro, O. M., Delgado Cordova, M., Ramirez Pastor, N., Mendoza Puza, G. M., \& Garcia Oyola, A. E. (2018). Violencia extrema contra la mujer y feminicidio en el Perú.

Trujillo Cristoffanini, M. (2019). Misoginia y violencia hacia las mujeres: dimensiones simbolicas del genero y del patriarcado. 0 .

Vaiz Bonifaz, R. G., \& Nakano, A. M. S. (2004). La violencia intrafamiliar, el uso de drogas en la pareja, desde la perspectiva de la mujer maltratada. Revista LatinoAmericana de Enfermagem, 12(spe), 433-438. https://doi.org/10.1590/S010411692004000700020

Vargas Murga, H. (2017). Violencia contra la mujer infligida por su pareja y su relación con la salud mental de los hijos adolescentesVargas Murga, H. (2017). Violencia contra la mujer infligida por su pareja y su relación con la salud mental de los hijos adolescentes. Revista Me. Revista Medica Herediana, 28(1), 48-58. https://doi.org/10.20453/RMH.V28I1.3074 